\title{
A visual survey for small non-stellar objects in the galactic plane on POSS II
}

\author{
Results of nineteen fields at $115^{\circ} \lesssim \ell \lesssim 157^{\circ}$ \\ R. Weinberger ${ }^{1}$, M. Gajdošík ${ }^{1}$, and C. $\operatorname{Zanin}^{1,2}$ \\ 1 Institut für Astronomie der Leopold-Franzens-Universität Innsbruck, Technikerstraße 25, A-6020 Innsbruck, Austria \\ 2 Dipartimento di Astronomia, Universitá di Padova, vicolo dell’Osservatorio 5, I-35122 Padova, Italy
}

Received March 8; accepted March 18, 1999

\begin{abstract}
A systematic search by microscope for nonstellar objects on POSS II R film copies in a part of the northern galactic plane has led to the detection of about 3500 objects. The vast majority are obscured galaxies, most of which are new. We present coordinates and optical diameters of these galaxy candidates, list coincidences with objects in optical and infrared catalogues and discuss their distribution along the galactic plane ${ }^{1}$.
\end{abstract}

Key words: catalogs - $(I S M)$ : dust, extinction Galaxy: structure

\section{Introduction}

In the era of digitized sky surveys and of various methods to automatically tell galaxies from stars, a visual examination of photographic plates or film copies might easily be considered to be obsolete. However, at low galactic latitudes it becomes quite difficult to distinguish the often very dim and small galaxies from stars and other diffuse objects. This implies that an automatic detection of galaxies is severely hampered since one would be forced to check most candidates visually. As a consequence, visual surveys should be given preference - provided they are carried out by persons with appropriate experience of this type of work.

We have demonstrated that useful information can be obtained by visually inspecting photographic prints; particularly, we could gain experience during optical searches for galaxies in the "zone of avoidance" (ZOA) of our Galaxy (e.g. Weinberger 1980; Weinberger 1995; Seeberger

Send offprint requests to: $\mathrm{R}$. Weinberger

1 The catalogue is available in electronic form only at the CDS via anonymous ftp to cdsarc.u-strasbg.fr (130.79.128.5) or via http://cdsweb.u-strasbg.fr/Abstract.html et al. 1996; Saurer et al. 1997). Extragalactic research in the $\mathrm{ZOA}$ is, for almost one decade, a quite booming area. A flourishing identification industry has led to the discovery of many thousand mainly optically identified extragalactic objects in a zone that was, by Hubble (1934, found to be practically devoid of galaxies. This development was and is, from a purely scientific point of view, triggered by several developments. One is the discovery of large-scale structures in the Universe and the insight that these structures will, of course, not come to a halt when approaching the ZOA. A second are the discussions about the Great Attractor that was suspected to be located at low galactic latitudes. Third, scientists also take the pick out of the bunch by the discovery and investigation of highly obscured (massive) nearby galaxies (e.g. Kraan-Korteweg et al. 1994; Huchtmeier et al. 1995).

Due to the favorable plate material (fine grain emulsions and considerable deepness), optical surveys for galaxies were particularly promising for the southern hemisphere. Major surveys there are those performed by Kraan-Korteweg (1996) and Saito et al. (1990), Saito et al. (1991) and Yamada et al. (1993).

In the northern hemisphere, due to the fact that only a small fraction of the new deep POSS II atlas was available up to the mid-nineties, the POSS I-E (red-sensitive) prints - less deep and having worse resolution compared to the southern plates or films - were used for analogous searches. Now, almost all of the POSS II film copies are available, and visual inspections on this excellent material should consequently result in the detection of many more galaxies. This prompted us to examine a promising part of the northern ZOA for non-stellar objects, the results of which are presented in this paper. 
Table 1. The fields of POSS II searched for our program

\begin{tabular}{c|ccccc}
\hline No. & Field & $\begin{array}{c}\text { Decl. } \\
(1950)\end{array}$ & R.A. & $\ell$ & $b$ \\
\hline 1 & 78 & $+65^{\circ}$ & $00^{\mathrm{h}} 00^{\mathrm{m}}$ & 117.8 & +2.9 \\
2 & 79 & $+65^{\circ}$ & $00^{\mathrm{h}} 44^{\mathrm{m}}$ & 122.5 & +2.4 \\
3 & 80 & $+65^{\circ}$ & $01^{\mathrm{h}} 28^{\mathrm{m}}$ & 127.1 & +2.7 \\
4 & 81 & $+65^{\circ}$ & $02^{\mathrm{h}} 12^{\mathrm{m}}$ & 131.6 & +3.8 \\
5 & 82 & $+65^{\circ}$ & $02^{\mathrm{h}} 56^{\mathrm{m}}$ & 135.9 & +5.6 \\
6 & 111 & $+60^{\circ}$ & $00^{\mathrm{h}} 00^{\mathrm{m}}$ & 116.9 & -2.0 \\
7 & 112 & $+60^{\circ}$ & $00^{\mathrm{h}} 38^{\mathrm{m}}$ & 121.6 & -2.6 \\
8 & 113 & $+60^{\circ}$ & $01^{\mathrm{h}} 16^{\mathrm{m}}$ & 126.4 & -2.4 \\
9 & 114 & $+60^{\circ}$ & $01^{\mathrm{h}} 54^{\mathrm{m}}$ & 131.1 & -1.6 \\
10 & 115 & $+60^{\circ}$ & $02^{\mathrm{h}} 32^{\mathrm{m}}$ & 135.6 & -0.1 \\
11 & 116 & $+60^{\circ}$ & $03^{\mathrm{h}} 10^{\mathrm{m}}$ & 139.8 & +2.1 \\
12 & 117 & $+60^{\circ}$ & $03^{\mathrm{h}} 48^{\mathrm{m}}$ & 143.7 & +4.8 \\
13 & 154 & $+55^{\circ}$ & $02^{\mathrm{h}} 45^{\mathrm{m}}$ & 139.2 & -3.9 \\
14 & 155 & $+55^{\circ}$ & $03^{\mathrm{h}} 18^{\mathrm{m}}$ & 143.3 & -1.6 \\
15 & 156 & $+55^{\circ}$ & $03^{\mathrm{h}} 51^{\mathrm{m}}$ & 147.2 & +1.2 \\
16 & 157 & $+55^{\circ}$ & $04^{\mathrm{h}} 24^{\mathrm{m}}$ & 150.6 & +4.4 \\
17 & 200 & $+50^{\circ}$ & $03^{\mathrm{h}} 30^{\mathrm{m}}$ & 147.6 & -4.8 \\
18 & 201 & $+50^{\circ}$ & $04^{\mathrm{h}} 00^{\mathrm{m}}$ & 151.4 & -1.8 \\
19 & 202 & $+50^{\circ}$ & $04^{\mathrm{h}} 30^{\mathrm{m}}$ & 154.9 & +1.6 \\
\hline
\end{tabular}

\section{Search criteria and method}

A total of 19 POSS II R film copies, each comprising a field size of about 40 square degrees, were scanned. The longitude range selected was $115^{\circ} \lesssim \ell \lesssim 157^{\circ}$ and in this interval all fields with centers at $b \lesssim 5^{\circ}$ were examined. Table 1 displays the list of fields.

The main reasons for selecting this longitude range were:

1. The Supergalactic Plane crosses the Galactic Plane at $\ell \sim 135^{\circ}$. The significance to probe the ZOA in a wide strip around this longitude is obvious - and is indicated by the fact that several bright galaxies (e.g. Maffei 1 and 2, IC 342) lie behind the galactic plane.

2. Beginning with $\ell \sim 160^{\circ}$, the surface density of galaxies in the ZOA sharply increases (Weinberger 1980) and the handling of data is thus made considerably more difficult; fortunately, a large set of data in the anticenter ZOA based on inspection of POSS I redsensitive plates already exists (Pantoja 1995; Seeberger et al. 1996; Saurer et al. 1997).

In an earlier paper (Seeberger et al. 1996) we have argued that red-sensitive surveys are the most suitable material for galaxy searches in the ZOA - hence our selection of the POSS II R films. The visual examination was done by one of us (MG) and a check of all objects together with the decision to finally accept or reject an object was done by RW. Individual films were scanned along overlapping strips of about $1 \mathrm{~cm}$ wide by use of a microscope at $16 \times$ magnification. Typically a film copy took about ten to twelve hours to scan.

Equatorial coordinates were determined using a highresolution digitizer and a suitable software developed at Innsbruck. The overall accuracy is about \pm 0.25 . Diameters of each galaxy candidate were determined using the microscope at $25 \times$ magnification and a glass plate with a $0.1 \mathrm{~mm}$ grating; e.g., the major-axis diameter is defined as the maximum extent of the galaxy as seen by the eye through the microscope and is specified in increments of 0.01 (however, the diameters are not this accurate - about \pm 0 ! 05 is probably more appropriate).

\section{Results and discussion}

\subsection{The catalogue}

In Table 2 we list our galaxy candidates in order of increasing galactic longitude. Due to the editorial policy of Astronomy and Astrophysics we publish this table, which comprises 58 print pages, in electronic form. Thus, only a sample page (the first page of Table 2 ) is given here.

The galaxy designations follow the IAU recommendation for the nomenclature of new objects:

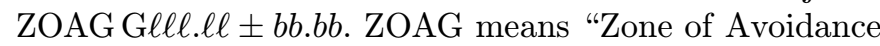
Galaxy", G stands for galactic coordinates, and $\ell \ell \ell . \ell \ell$ and $\pm b b . b b$ are galactic longitude and latitude, respectively.

In Col. 1 the designation of the galaxies is given. For reasons of brevity the prefix ZOAGG is omitted. In a few cases, this designation is not unambiguous, i.e. 2 (or more) very closely located galaxies could not be separated. For this we add a suffix "a" for the object with the smaller (smallest) right ascension. If the right ascensions are practically the same, then the object with the smallest declination is given the suffix "a", then "b" for the next higher declination and so forth. If both R.A. and Dec. are almost identical, then (and only then) the more optically extended one of the objects gets the suffix "a". Columns 2 and 3 give the equatorial coordinates for epoch 1950.0, Cols. 4 and 5 for epoch 2000.0 (Julian). In Col. 6 we present maximum and minimum diameters (in arcmin) measured from the POSS II R film copies. In the last column we list cross-identifications with galaxies taken from the NASA Extragalactic Database (NED), and with IRAS point sources taken from SIMBAD. For crossidentifications with the IRAS PSC catalogue we used our above-given positional uncertainty and checked whether our optical error bars fell within the IRAS uncertainty ellipse or not.

\subsection{The distribution}

In Fig. 1 (top) all our galaxy candidates are plotted, and in Fig. 1 (bottom) the $100 \mu \mathrm{m}$ IRAS surface brightness is shown. From Fig. 1 (top), several distinctive features are obvious: First, the ZOA does not appear to be prominent (particularly if compared, e.g., with Fig. 3 in Seeberger et al. (1994) that is based on galaxy candidates found on POSSI) - there are numerous galaxy candidates even 
Table 2. The catalogue

\begin{tabular}{|c|c|c|c|c|c|c|}
\hline ZOAG G & $\alpha(1950)$ & $\delta(1950)$ & $\alpha(2000)$ & $\delta(2000)$ & $\varnothing R$ & cross id. \\
\hline $113.83-3.63$ & 233939.0 & 574357 & 234203.8 & 580036 & 0.240 .14 & \\
\hline $113.92-2.93$ & 233852.9 & 582603 & $2341 \quad 17.1$ & 584241 & 0.150 .13 & \\
\hline $114.47-4.51$ & 234551.3 & 570237 & 234819.2 & 571918 & 0.220 .19 & \\
\hline $114.56-3.94$ & 234531.4 & 573650 & 234759.0 & 575331 & 0.110 .10 & \\
\hline $114.56-4.46$ & 234626.9 & 570642 & 234855.0 & 572323 & 0.210 .08 & \\
\hline $114.64-4.20$ & 234631.6 & 572308 & 234859.7 & 573949 & 0.460 .43 & \\
\hline $114.67-4.59$ & 234724.3 & 570049 & 234952.9 & 571730 & 0.630 .15 & \\
\hline $114.83-2.61$ & 234500.2 & 585819 & 234727.2 & 591459 & 0.100 .08 & \\
\hline $114.89-4.63$ & 234904.6 & 570120 & 235134.0 & 571801 & 0.110 .10 & \\
\hline $114.90-4.63 \mathrm{a}$ & 234907.2 & 570128 & 235136.6 & 571809 & 0.110 .08 & \\
\hline $114.90-4.63 \mathrm{~b}$ & 234908.3 & 570128 & 235137.7 & 571809 & 0.100 .08 & \\
\hline $115.02-3.95$ & 234851.1 & 574318 & 235120.2 & 575959 & 0.100 .08 & \\
\hline $115.23-4.39$ & 235107.9 & 572012 & 235338.1 & 573654 & 0.110 .10 & \\
\hline $115.28-4.00$ & 235051.7 & 574326 & 235321.8 & 580008 & 0.100 .10 & \\
\hline $115.32-3.97$ & 235104.8 & 574606 & 235335.0 & 580248 & 0.070 .07 & \\
\hline $115.43+5.10:$ & 233155.1 & 663354 & 233410.6 & 665029 & 0.110 .08 & IRAS $23319+6633$ \\
\hline $115.56-4.53$ & 235344.4 & 571618 & 235615.8 & 573300 & 0.110 .10 & \\
\hline $115.75-4.03$ & 235420.8 & 574746 & 235652.5 & 580428 & 0.100 .08 & \\
\hline $115.82-3.70$ & 235416.1 & 580827 & 235647.7 & 582509 & 0.150 .11 & \\
\hline $115.84-2.10$ & 235147.5 & 594229 & 235417.7 & 595911 & 0.110 .11 & \\
\hline $115.93+0.14:$ & 234819.7 & 615416 & 235047.7 & 621057 & 0.110 .08 & \\
\hline $115.95-4.34$ & 235615.9 & 573217 & 235848.5 & 574859 & 0.100 .07 & \\
\hline $116.12-3.66$ & 235627.3 & 581418 & 235859.9 & 583100 & 0.200 .18 & \\
\hline $116.15-4.73$ & 235818.0 & 571156 & 000051.5 & 572838 & 0.210 .10 & \\
\hline $116.36-4.59$ & 235935.8 & 572234 & 000209.9 & 573916 & 0.130 .11 & \\
\hline $116.36-4.89$ & 235959.2 & 570445 & 000233.5 & 572127 & 0.110 .09 & \\
\hline $116.37+5.45$ & 234006.5 & 670925 & 234227.2 & 672604 & 0.100 .08 & \\
\hline $116.42-3.53$ & 235828.5 & 582542 & 000102.1 & 584224 & 0.100 .07 & \\
\hline $116.45-4.05$ & 235928.4 & 575529 & 000202.5 & 581211 & 0.090 .07 & \\
\hline $116.48-4.64$ & 000033.7 & 572056 & 000308.3 & 573738 & 0.090 .08 & \\
\hline $116.53-4.32$ & 000027.9 & 574012 & 000302.4 & 575654 & 0.130 .12 & \\
\hline $116.56-1.23$ & 235559.8 & 604218 & 235832.1 & 605900 & 0.570 .15 & \\
\hline $116.57-2.39$ & 235754.4 & 593400 & 000027.7 & 595042 & 0.110 .09 & NVSS J000027+595048 \\
\hline $116.58-4.89$ & 000136.9 & 570714 & 000411.9 & 572356 & 0.200 .08 & \\
\hline $116.59-5.14$ & 000201.8 & 565240 & 000437.0 & 570922 & 0.140 .11 & \\
\hline $116.62+0.35$ & 235344.2 & 621554 & 235615.1 & 623236 & 0.240 .07 & \\
\hline $116.64-3.54 \mathrm{a}$ & 000010.7 & 582718 & 000245.1 & 584400 & 0.160 .09 & \\
\hline $116.64-3.54 \mathrm{~b}$ & $\begin{array}{lll}00 & 00 & 10.9\end{array}$ & 582732 & 000245.3 & 584414 & 0.110 .08 & \\
\hline $116.71-3.35$ & 000026.5 & 583923 & 000301.1 & 585605 & 0.150 .12 & \\
\hline $116.76-4.05$ & 000145.6 & 575849 & 000420.8 & 581531 & 0.090 .09 & \\
\hline $116.82+5.48$ & 234425.1 & 671828 & 234648.7 & 673508 & 0.090 .08 & \\
\hline $116.84-4.06$ & 000223.9 & 575848 & $00 \quad 0459.4$ & 581530 & 0.100 .08 & \\
\hline $116.86-2.04$ & 235940.0 & 595810 & $\begin{array}{lll}00 & 02 & 14.2\end{array}$ & 601452 & 0.190 .15 & \\
\hline $116.91-4.12$ & 000256.6 & 575618 & 000532.3 & 581300 & 0.100 .09 & \\
\hline $116.99-3.32$ & 000229.4 & 584404 & 000505.0 & 590046 & 0.110 .10 & \\
\hline $116.99-4.59$ & 000411.4 & 572912 & 000647.7 & 574554 & 0.110 .08 & \\
\hline $117.08-5.19$ & 000533.5 & 565445 & $0008 \quad 10.3$ & 571127 & 0.180 .12 & \\
\hline $117.12-5.08$ & 000544.4 & 570136 & 000821.3 & 571818 & 0.150 .07 & \\
\hline $117.17-4.51$ & 000523.5 & 573601 & 000800.3 & 575243 & 0.360 .07 & \\
\hline $117.18-4.50$ & 000528.2 & 573641 & 000805.1 & 575323 & $0.14 \quad 0.11$ & \\
\hline $117.26-1.39$ & 000147.7 & 604046 & 000423.0 & 605728 & 0.110 .10 & \\
\hline $117.26-5.08$ & 000644.5 & 570254 & 000921.9 & 571936 & 0.070 .07 & \\
\hline $117.33-4.54$ & 000637.6 & 573554 & 000915.0 & 575236 & 0.130 .10 & \\
\hline $117.39-5.24$ & 000753.2 & 565441 & 001031.1 & 571122 & 0.180 .11 & \\
\hline $117.41-4.91$ & 000740.2 & 571423 & 001018.0 & 573104 & 0.090 .08 & \\
\hline $117.42-4.85$ & 000738.8 & 571750 & 001016.6 & 573431 & 0.080 .07 & \\
\hline $117.43-5.21$ & 000806.5 & 565706 & 001044.5 & 571347 & 0.100 .08 & \\
\hline
\end{tabular}



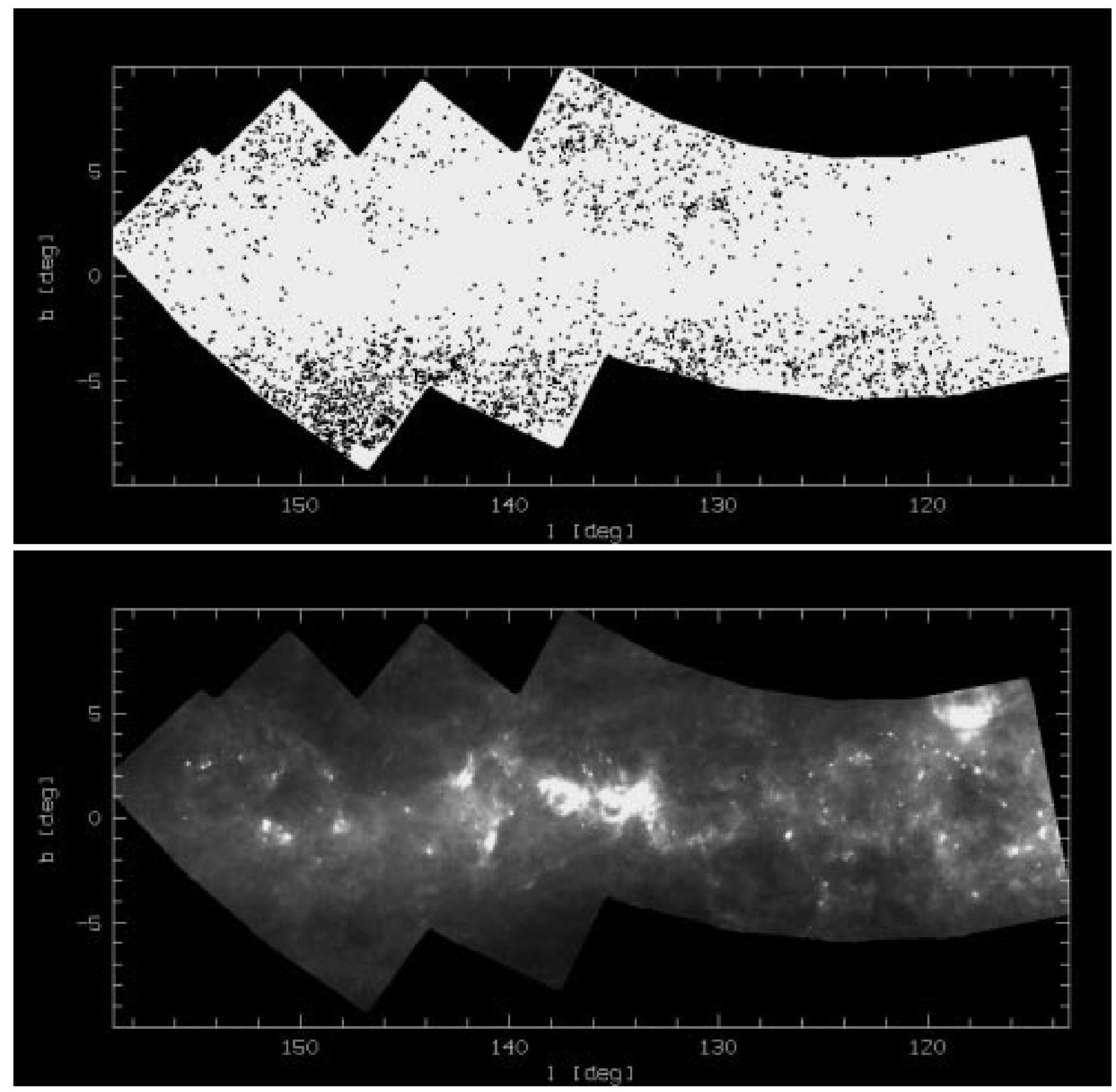

Fig. 1. Top: Distribution of the galaxies in galactic coordinates. The south-west corners of the fields lack objects due to an area containing sensitometer spots. Bottom: A grayscale map of IRAS $100 \mu \mathrm{m}$ intensities (white corresponds to bright emission). Regions outside the surveyed area are black

very close to the galactic equator. Second, the number of galaxies at negative galactic latitudes is much larger than at positive ones. Third, there are only very few objects at $\ell \lesssim 125^{\circ}$ at positive latitudes. The distribution of galaxies can be influenced in two main ways, i.e. it can be of i) galactic (i.e. foreground) origin and/or it can ii) reflect the true distribution, like concentrations (clusters) of galaxies. The former origin might, in part, be tested by comparing the galaxy distribution with maps of (infrared emitting) dust clouds. As can easily be seen from a comparison of Fig. 1 (top) with 1 (bottom), regions of intense IR-emission are generally avoided by galaxies; however, in a few cases there appear to be positional coincidences that must be examined, since e.g. small red nebulae in star forming regions sometimes mimic the appearance of obscured galaxies, since they cannot be easily eliminated 


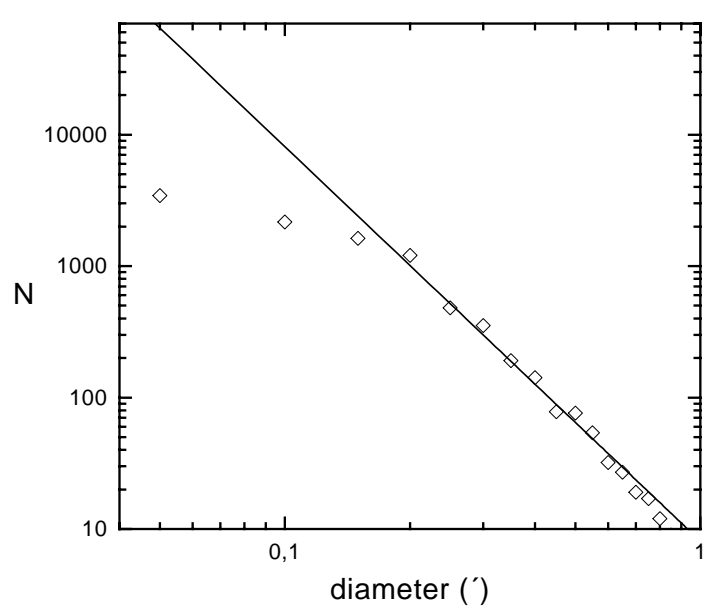

Fig. 2. Squares: logarithm of the total number of galaxies down to a given angular diameter versus the logarithm of this very diameter. The line corresponds to the expected slope of -3

even if checking plates taken in the photographic infrared (like POSS II IR).

\subsection{Completeness of the catalogue}

The limit of completeness of the catalogue may be estimated by plotting the number of galaxies with diameters larger or equal than a certain value against this value. For a homogeneous spatial distribution of galaxies having all the same typical linear dimension $N \sim d^{-3}$ applies. Figure 2 shows that our sample of galaxies starts to seriously deviate from completeness at angular diameters of $d \leq 0.2$. Similar optical surveys performed at the POSS I plates were almost complete for $d \geq 0$.4 (Seeberger et al. 1996; Lercher et al. 1996). Certainly our better limit of completeness is due to the higher quality of the POSS II plates, especially thanks to a finer grain emulsion. The figure also indicates that we missed roughly $73 \%$ of galaxies with diameters less than 0.1 . When comparing to Seeberger et al. (1996) who missed roughly $90 \%$ of this sized galaxies one can guess that $\approx 3 \times$ more galaxy candidates will be selected on POSS II R plates than on POSS I E.

Nevertheless, we were able to directly compare the total number of galaxy candidates selected in optical surveys on POSS I E and POSS II R plates since a substantial portion of our region was already surveyed on the older plates (Lercher et al. 1996; Saurer et al. 1997). By counting the galaxies in the intersection of both surveys we obtained 674 and 2072 galaxies detected on POSS I and POSS II, respectively. Thus, we may roughly state that on the "new" POSS R plates (i.e. POSS II) $3.1 \times$ more galaxy candidates are selected than on the "old" ones.

\subsection{IRAS two-colour diagram}

Out of our sample of 3455 galaxies 144 have an IRAS counterpart (as results from the check of the NED and

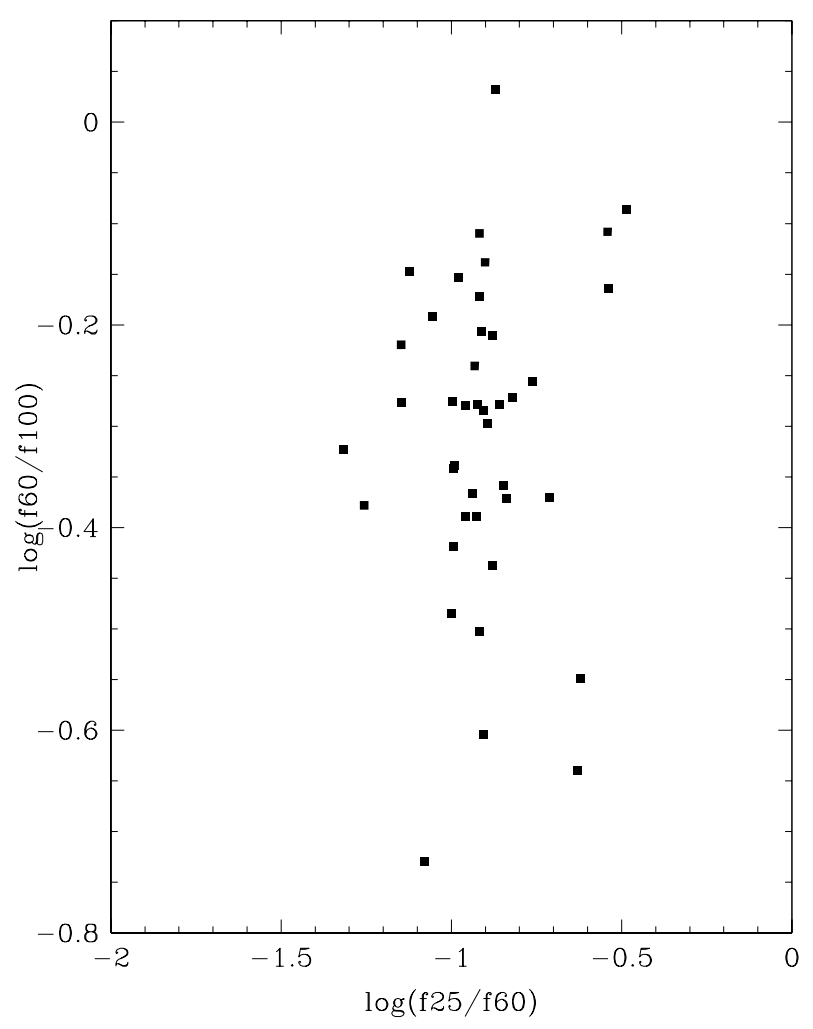

Fig. 3. IRAS two-colour diagram for 43 galaxies with fluxes of a good quality

SIMBAD catalogue), i.e. 0.26 galaxies per square degree. This value is not larger than that found for POSS I surveys (Seeberger et al. 1996 and references therein). Similarly to Seeberger et al. (1996) we plotted a two-colour diagram of the IRAS point sources for those with a flux quality $\geq 2$ (see Fig. 3). From our sample 43 sources fulfilled this condition, giving almost identical mean colours as quoted in Seeberger et al. (1996).

Acknowledgements. This work was supported by the "Jubiläumsfonds der Österreichischen Nationalbank" under project No. 5776. We would also like to thank S. Kimeswenger, W. Marchiotto and S. Temporin for various help.

This research has made use of the NASA/IPAC Extragalactic Database (NED) which is operated by the Jet Propulsion Laboratory, Caltech, under contract with the National Aeronautics and Space Administration.

We have made use of the SIMBAD database which is managed by the Centre de Données astronomiques de Strasbourg (CDS).

\section{References}

Hubble E., 1934, ApJ 79, 8

Huchtmeier W.K., Lercher G., Seeberger R., Saurer W., Weinberger R., 1995, A\&A 293, L33

Kraan-Korteweg R.C., Woudt P.A., Cayatte V., et al., 1996, Nat 379, 519

Kraan-Korteweg R.C., Loan A.J., Burton W.B., et al., 1994, Nat 372,77 
Lercher G., Kerber F., Weinberger R., 1996, A\&AS 117, 369

Pantoja C.A., 1995, Ph.D. thesis, Univ. of Oklahoma

Saito M., Ohtani H., Asomuna A., et al., 1990, PASJ 42, 603

Saito M., Ohtani H., Baba A., et al., 1991, PASJ 43, 449

Seeberger R., Saurer W., Weinberger R., 1996, A\&AS 117, 1

Seeberger R., Saurer W., Weinberger R., Lercher G., 1994,

Proc. of the 4th DAEC-meeting on: Unveiling large-scale structures behind the Milky Way, p. 81

Saurer W., Seeberger R., Weinberger R., 1997, A\&AS 126, 247

Weinberger R., 1980, A\&AS 40, 123

Weinberger R., 1995, PASP 107, 58

Yamada T., Takata T., Djamaluddin T., et al., 1993, ApJS 89, 57 